\title{
COLON
}

\section{In vivo imaging of cellular proliferation in colorectal cancer using positron emission tomography}

\author{
D L Francis, A Freeman, D Visvikis, D C Costa, S K Luthra, M Novelli, I Taylor, P J Ell
}

See end of article for authors' affiliations .....................

Correspondence to: D Francis, Institute of Nuclear Medicine, Middlesex Hospital Mortimer St, London, W1T 3AA, UK; daren.francis@ ucl.ac.uk

Accepted for publication 8 July 2003
Background and aims: Positron emission tomography (PET) using ${ }^{18} \mathrm{~F}$ labelled 2-fluoro-2-deoxy-Dglucose $\left({ }^{18} \mathrm{FDG}\right)$ is an established imaging tool, although the recent development of a biologically stable thymidine analogue [18F] 3'-deoxy-3-fluorothymidine $\left({ }^{18} \mathrm{FLT}\right)$ has allowed PET to image cellular proliferation by utilising the salvage pathway of DNA synthesis. In this study, we have compared uptake of ${ }^{18} \mathrm{FLT}$ and ${ }^{18} \mathrm{FDG}$ with MIB-1 immunohistochemistry to evaluate the role of PET in quantifying in vivo cellular proliferation in colorectal cancer (CRC).

Patients and methods: Patients with resectable, primary, or recurrent CRC were prospectively studied. Thirteen lesions from 10 patients (five males, five females), median age 68 years (range 54-87), were evaluated. Patients underwent ${ }^{18} \mathrm{FDG}$ and ${ }^{18} \mathrm{FLT}$ PET scanning. Tracer uptake within lesions was quantified using standardised uptake values (SUVs). Histopathological examination and MIB-1 immunohistochemistry were performed on all lesions, and proliferation quantified by calculating a labelling index (\% of MIB-1 positively stained nuclei within 1500 tumour cells).

Results: Histology confirmed adenocarcinoma in 12 of 13 lesions; the remaining lesion was reactive. All eight extrahepatic lesions were visualised using both ${ }^{18} \mathrm{FLT}$ and ${ }^{18} \mathrm{FDG}$. Three of the five resected liver metastases were also avid for ${ }^{18} \mathrm{FLT}$ and showed high proliferation, while the remaining two lesions which demonstrated no uptake of ${ }^{18} \mathrm{FLT}$ had correspondingly very low proliferation. There was a statistically significant positive correlation $(r=0.8, p<0.01)$ between SUVs of the tumours visualised with ${ }^{18} \mathrm{FLT}$ and the corresponding MIB-1 labelling indices. No such correlation was demonstrated with ${ }^{18} \mathrm{FDG}$ avid lesions $(r=0.4)$.

Conclusions: ${ }^{18} \mathrm{FLT}$ PET correlates with cellular proliferation markers in both primary and metastatic CRC. This technique could provide a mechanism for in vivo grading of malignancy and early prediction of response to adjuvant chemotherapy.
$\mathrm{T}$ he success of positron emission tomography (PET) in oncological imaging is based on the knowledge that malignancy leads to an alteration in cellular biochemical reactions. Synthesis of positron emitting analogues from molecules of organic matter can demonstrate these biochemical processes, thus highlighting the presence of malignancy. ${ }^{18} \mathrm{~F}$ labelled 2 -fluoro-2-deoxy-D-glucose $\left({ }^{18} \mathrm{FDG}\right)$ is the most commonly used PET tracer and its utility relies on the increased glucose consumption of malignant cells. The combination of PET and ${ }^{18}$ FDG is an established imaging tool which has made a significant impact in colorectal cancer (CRC). ${ }^{1-9}$ Conversion of this tracer into a monophosphate (FDG-6-PO4) by the enzyme hexokinase leads to intracellular trapping, and following radioactive decay its distribution can be mapped in vivo.

The unique ability of this technique to image cellular biology in vivo has led to extensive research into the potential role of PET in the non-invasive assessment of cellular proliferation in various solid tumours. ${ }^{10-16}$

Previous methods of quantifying cell cycle activity in colorectal cancer have included incorporation of radiolabelled nucleotides and flow cytometry. While flow cytometry has shown a relationship between high $\mathrm{S}$ phase fractions and increased probability of recurrence and diminished survival, ${ }^{17-19}$ the more recent use of immunohistochemistry using the MIB-1 antibody (which recognises the cell cycle associated Ki-67 protein) has allowed an easier way to assess proliferation and is now more commonly used in routine clinical practice. Expression of the Ki-67 protein occurs during the Gl phase of the cell cycle, increasing during the cycle, and then rapidly declines after mitosis, ${ }^{20}$ allowing assessment of virtually all cycling cells regardless of their stage in the cell cycle.

The relationship of FDG to proliferative activity has been examined both in vitro and in vivo with varying results. Higashi and colleagues ${ }^{21}$ demonstrated in ovarian adencarcinoma cell lines that utilisation of glucose by tumour cells is not a reflection of cell proliferation but is strongly related to the number of viable tumour cells. Buck et al attempted to use FDG PET in vivo to estimate proliferative activity as a means of differentiating pancreatic cancer from chronic active pancreatitis but found no correlation between FDG uptake and cellular proliferation using MIB-1 immunostaining. ${ }^{22}$

The recent development of the biologically stable thymidine analogue[18F] 3 '-deoxy-3-fluorothymidine $\left({ }^{18} \mathrm{FLT}\right)^{23}$ has allowed PET to visualise a different biochemical process. This is achieved by targeting the salvage pathway of DNA synthesis, and hence the images appear more directly related to cellular proliferation. Accumulation of ${ }^{18}$ FLT in proliferating cells occurs by virtue of the enzyme thymidine kinase 1 (TK), which is closely linked to the $S$ phase of the cell cycle. This salvage pathway enzyme fluctuates with DNA synthesis, the activity being high in proliferating and malignant cells

\footnotetext{
Abbreviations: PET, positron emission tomography; $C T$, computed tomography; ${ }^{8}{ }^{8} \mathrm{FDG},{ }^{18} \mathrm{~F}$ labelled 2-fluoro-2-deoxy-D-glucose; ${ }^{18} \mathrm{FLT}$, ${ }^{18} \mathrm{~F}$ labelled 3 '-deoxy-3-fluorothymidine; CRC, colorectal cancer; TK' thymidine kinase 1; SUV, standardised uptake value; ROI, regions of interest; H\&E, haematoxylin and eosin; TBS, Tris buffered saline; LI, labelling index
} 
and low or absent in quiescent cells. ${ }^{24}$ High TK levels have been shown to be an important risk factor in patients with breast cancer $^{25}$ and in addition in vitro studies have demonstrated a positive correlation between FLT uptake, cell growth, and TK activity. ${ }^{26}$

The aim of this prospective study was thus to compare tumour uptake by both ${ }^{18}$ FLT and ${ }^{18}$ FDG PET and investigate their correlation with cellular proliferation using MIB-1 immunohistochemistry. To our knowledge this is the first study evaluating the role of PET in quantifying in vivo cellular proliferation in CRC.

\section{PATIENTS AND METHODS \\ Patient selection}

Ten patients (five males, five females), median age 68 years (range 54-87), with 13 resectable primary or recurrent colorectal cancers, were recruited at diagnosis from local surgical clinics. Demographics are given in table 1. Written informed consent was obtained following approval from the Local Research Ethics Committee and the Administration of Radioactive Substances Advisory Committee. All patients underwent whole body ${ }^{18}$ FDG scanning followed by an ${ }^{18}$ FLT scan (median intervening period 5 days (range 1-19)).

\section{Imaging}

${ }^{18}$ FDG was prepared using a modification of the technique described by Hamacher and colleagues ${ }^{27}$ while ${ }^{18}$ FLT was prepared according to the method of Cleij and colleagues. ${ }^{28}$ Radiochemical purity was $>99 \%$ and $>95 \%$ for ${ }^{18} \mathrm{FDG}$ and ${ }^{18}$ FLT, respectively. All patients were asked to fast for six hours prior to scanning, and blood glucose levels were checked using a glucometer (Glucometer Elite; Bayer Pharmaceuticals, Newbury, Berkshire, UK) prior to injection of the tracers. All patients were normoglycaemic with a mean blood glucose concentration of $5.4 \mathrm{mmol} / \mathrm{l}$ (SD 0.5) for ${ }^{18} \mathrm{FDG}$ and $4.6 \mathrm{mmol} / \mathrm{l}$ (SD 0.8 ) for ${ }^{18}$ FLT. Mean time of data acquisition post injection was 57 minutes (SD 10) and 62 minutes (SD 12) for ${ }^{18} \mathrm{FDG}$ and ${ }^{18}$ FLT, respectively. The timing of ${ }^{18}$ FLT scanning in patients with this disease was determined by previous work carried out by our group using time-activity curves. ${ }^{29}$ Mean injected activity was $371 \mathrm{MBq}$ (SD 24) and $351 \mathrm{MBq}$ (SD 52), respectively. All data were acquired using the Discovery LS PET/computed tomography (CT) combined scanner (GE medical systems, Milwaukee, USA). PET emission datasets were attenuation corrected utilising the CT capability of the hybrid scanner. ${ }^{30}$ Spiral CT scans were acquired using a speed of rotation and couch movement of $0.8 \mathrm{~s}$ and $22.5 \mathrm{~mm}$ per rotation, respectively, with a slice thickness of $5 \mathrm{~mm}$. CT images were subsequently converted to maps of PET attenuation coefficients using a bilinear transformation. Transaxial emission images of $4.3 \times 4.3 \times 4.25 \mathrm{~mm}^{3}$ (matrix size $128 \times 128 \times 35$ ) were reconstructed using ordered subsets expectation maximisation with two iterations and 28 subsets.

For each patient, regions of interest (ROI) were drawn over the tumour and standardised uptake values (SUVs) were obtained for both tracers. SUVs were calculated using the average tumour activity concentration $(A)$ in $\mathrm{kBq} / \mathrm{ml}$, the patient's body weight $(W)$ in $\mathrm{kg}$, and the injected activity $\left(A_{\text {inj }}\right)$ in kBq:

$$
\mathrm{SUV}=(A \times W) / A_{\text {inj }}
$$

The average SUV of five consecutive slices was obtained. The slices selected comprised the one with the maximum count density over each ROI and the four immediately adjacent slices.

\section{Immunohistochemistry}

Routine histological examination was performed on $4 \mu \mathrm{m}$ thick haematoxylin and eosin (H\&E) stained sections. Each specimen was sampled in a routine manner for diagnostic purposes in accordance with established Royal College of Pathology clinical guidelines. Immunohistochemistry for Ki-67 using the MIB-1 antibody was then performed on routine diagnostic blocks using $3 \mu \mathrm{m}$ thick sections cut from paraffin blocks and dried overnight at $60^{\circ} \mathrm{C}$. Sections were taken from xylene (two changes), through graded alcohols $(100 \%$ and $70 \%)$ to water. Antigen retrieval was performed by pressure cooking for two minutes in a conventional $15 \mathrm{lb}$ pressure cooker. Slides were then rinsed in $0.05 \%$ Tween 20 in Tris buffered saline (TBS/Tween). Endogenous peroxidase activity was blocked for 10 minutes using a commercially available peroxidase blocking solution (Dako, Ely, Cambridgeshire, UK). Primary mouse monoclonal antibody (MIB-1 (Dako) diluted 1/50 in TBS, pH 7.4) was applied for 60 minutes at room temperature.

Sections were rinsed in TBS/Tween and secondary antibody (goat antirabbit/mouse; Dako) was applied for 30 minutes. Sections were then rinsed in TBS/Tween and streptavidinhorseradish peroxidase (Dako) was applied for 30 minutes. Sections were again rinsed in TBS/Tween and diaminobenzidene used as a chromogen. A haematoxylin counter stain was applied.

Sections were dehydrated through graded alcohols (70\% and $100 \%$ ), cleared in xylene (two changes), and coverslipped with DPX mountant.

\section{Examination of sections}

Sections of tumour were identified on the H\&E slides and the corresponding MIB-1 sections were examined independently

Table 1 Patient characteristics, including calculated standardised uptake values and labelling indices for the respective lesions

\begin{tabular}{|c|c|c|c|c|c|c|c|}
\hline Patient No & Sex & Age $(y)$ & Weight (kg) & Resected lesions & ${ }^{18} \mathrm{FDG}$ (SUV) & ${ }^{18} \mathrm{FLT}$ (SUV) & LI (\% MIB-1) \\
\hline 1 & $M$ & 87 & 72 & Primary (asc colon) & 9.2 & 3.5 & 69 \\
\hline \multirow[t]{2}{*}{2} & M & 66 & 82 & Peritoneal recurrence & 7.6 & 3.3 & 60 \\
\hline & & & & Peritoneal lesiont & 7.8 & - & 23 \\
\hline \multirow[t]{2}{*}{3} & $\mathrm{~F}$ & 78 & 54 & Liver metastases $1^{*}$ & 5.5 & - & 19 \\
\hline & & & & Liver metastases $2^{*}$ & 11 & - & 26 \\
\hline \multirow[t]{2}{*}{4} & $\mathrm{~F}$ & 75 & 63 & Primary (rectal) & 9.6 & 4.8 & 70 \\
\hline & & & & Liver metastases & 3.7 & 9.1 & 87 \\
\hline 5 & M & 62 & 85 & Primary (caecal) & 9.7 & 5.5 & 77 \\
\hline 6 & $\mathrm{~F}$ & 60 & 58 & Liver metastases & 3.7 & 4.8 & 73 \\
\hline 7 & $M$ & 59 & 70 & Primary (trans colon) & 8.5 & 2.9 & 67 \\
\hline 8 & $\mathrm{~F}$ & 54 & 55 & Primary (rectal) & 5.9 & 1.4 & 69 \\
\hline 9 & $\mathrm{~F}$ & 67 & 95 & Peritoneal recurrence & 4.5 & 2.6 & 75 \\
\hline 10 & $M$ & 71 & 65 & Liver metastases & 4.7 & 6.1 & 87 \\
\hline
\end{tabular}

${ }^{18} \mathrm{FDG},{ }^{18} \mathrm{~F}$ labelled 2-fluoro-2-deoxy-D-glucose; ${ }^{18} \mathrm{FLT}, 3^{\prime}$-deoxy-3-fluorothymidine; SUVs, standardised uptake values; LI, labelling index. *Those lesions seen as avid for ${ }^{18} \mathrm{FDG}$ but not for ${ }^{18} \mathrm{FLT}$. tInflammatory lesions not demonstrating uptake for ${ }^{18} \mathrm{FLT}$. 
by two experienced observers, $\mathrm{A}$ and $\mathrm{B}$, blinded to the results of the scans.

Three MIB-1 stained sections of tumour were used for each lesion to account for tumour heterogeneity. Thirty high power fields ( $\times 40$ objectile lens) were examined and 50 tumour cells were assessed per field using a graticule, making a total of 1500 cells per case. A labelling index (LI) was calculated as the percentage of positive staining nuclei out of all nuclei examined. "Positive" nuclear staining was regarded as staining of the entire nucleus. Nuclei with no staining or nucleolar staining only was regarded as negative.

\section{Statistical analysis}

Interobserver reliability was assessed using Pearson's correlation coefficient, and linear regression analysis using the SPSS statistical package was employed to correlate uptake of both ${ }^{18}$ FLT and ${ }^{18}$ FDG with MIB-1.

\section{RESULTS}

Histopathology of all lesions $(n=13$, five primary, five liver metastases, and three peritoneal) confirmed adenocarcinoma in 12 of 13 cases. The remaining case was thought to be a malignant peritoneal recurrence by preoperative CT, ${ }^{18}$ FDG PET imaging, and at intraoperative evaluation but on microscopic histological examination was found to be an inflammatory lesion secondary to fat necrosis.

There was excellent interrater reliability between the two observers responsible for quantifying proliferation using MIB-1 immunohistochemistry (Pearson's correlation coefficient 0.9) (fig 1).

Quantification of tracer uptake in addition to the corresponding LIs can be seen in table 1 . All five primary tumours in addition to both malignant peritoneal lesions were visualised with increased focal uptake using both ${ }^{18}$ FLT and ${ }^{18}$ FDG. Three of five liver metastases were clearly seen to be avid for ${ }^{18} \mathrm{FLT}$, and all showed a corresponding high proliferation LI.

The three lesions (peritoneal inflammatory lesion and two liver metastases) which did not show increased uptake of ${ }^{18}$ FLT and were therefore not visualised using this tracer had corresponding low proliferation rates, as determined by the LIs, ranging from 19 to 26.

SUVs for those lesions seen with ${ }^{18}$ FDG ranged between 3.7 and 11 compared with a range of 1.4-9.1 for the 10 lesions visualised using ${ }^{18}$ FLT.

There was a statistically significant correlation $(r=0.8$, $\mathrm{p}<0.01$ ) between SUVs of the 10 tumours visualised with ${ }^{18}$ FLT and the corresponding LIs (fig 2). No such correlation

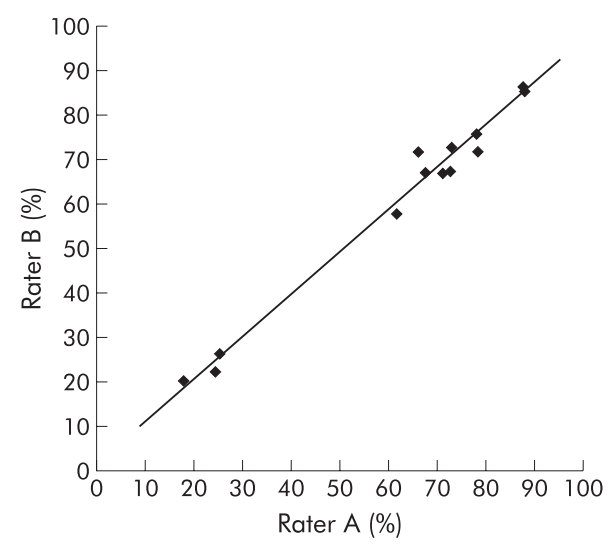

Figure 1 Interrater reliability between observers A and B, with a Pearson correlation coefficient of 0.9 .

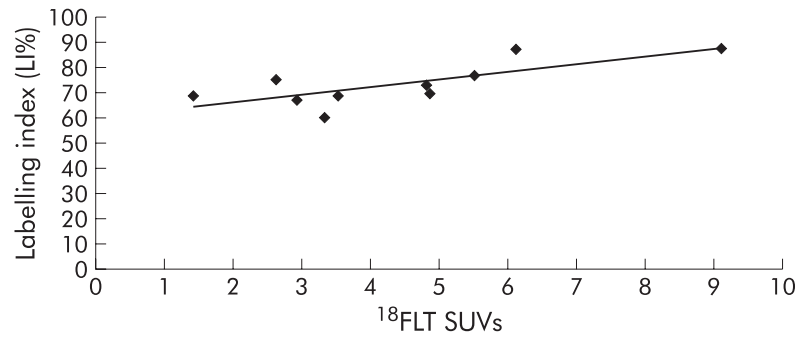

Figure 2 Linear regression analysis for those lesions seen with ${ }^{18} \mathrm{~F}$ labelled $3^{\prime}$-deoxy-3-fluorothymidine $\left({ }^{18} \mathrm{FLT}\right)(\mathrm{n}=10)$, correlating labelling indices (LIs) with standardised uptake values (SUVs) $(r=0.8)$.

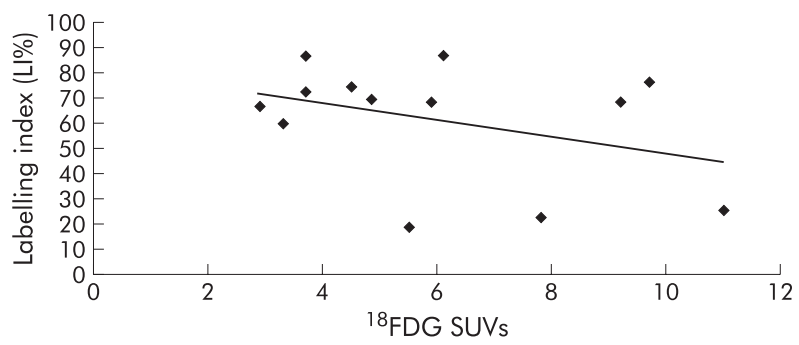

Figure 3 Linear regression analysis for those lesions seen with ${ }^{18} \mathrm{~F}$ labelled 2-fluoro-2-deoxy-D-glucose ( $\left.{ }^{8} \mathrm{FDG}\right)(\mathrm{n}=13)$, correlating labelling indices (Lls) with standardised uptake values (SUVs) $(r=0.4)$

$(r=0.4)$ was demonstrated in the case of ${ }^{18}$ FDG avid lesions (fig 3).

Differences in tracer uptake compared with macroscopic histology and MIB-1 immunohistochemistry are highlighted in fig 4 .

\section{DISCUSSION}

Our results suggest that ${ }^{18} \mathrm{FLT}$ reflects proliferative activity in CRC, as determined by MIB-1 staining. Lack of correlation between LIs and ${ }^{18}$ FDG supports previous evidence that ${ }^{18}$ FDG is not a specific marker of proliferation. ${ }^{21}{ }^{22}$ The lesions successfully imaged with ${ }^{18}$ FLT demonstrated high SUVs in addition to high LIs. On the other hand, those lesions not visualised by ${ }^{18}$ FLT were shown to have correspondingly low LIs.

It is known that glucuronidation of ${ }^{18}$ FLT within the liver leads to high hepatic background activity which may inhibit its diagnostic utility. ${ }^{31}$ This was exemplified by failure of ${ }^{18}$ FLT PET to show two of the five liver metastases against this high liver background. However, both of these lesions displayed low proliferation labelling indices (19\% and 26\%), suggesting relatively slow growth compared with the remaining three liver metastases (labelling indices of $87 \%$, $87 \%$, and $73 \%$ ). The fact that certain liver lesions are not seen with ${ }^{18}$ FLT may have prognostic implications in terms of the aggressiveness of these tumours.

The well documented problems of specificity due to uptake of ${ }^{18} \mathrm{FDG}$ by activated macrophages ${ }^{32-34}$ is also highlighted in this study by the peritoneal lesion thought to be malignant because of a high uptake of ${ }^{18}$ FDG. Microscopic examination of the entire lesion showed a florid inflammatory response and fat necrosis secondary to previous surgery, but no tumour. In contrast, ${ }^{18}$ FLT showed no increased uptake within this lesion, suggesting that this new tracer may have a role to play in improving the diagnostic specificity of PET. 

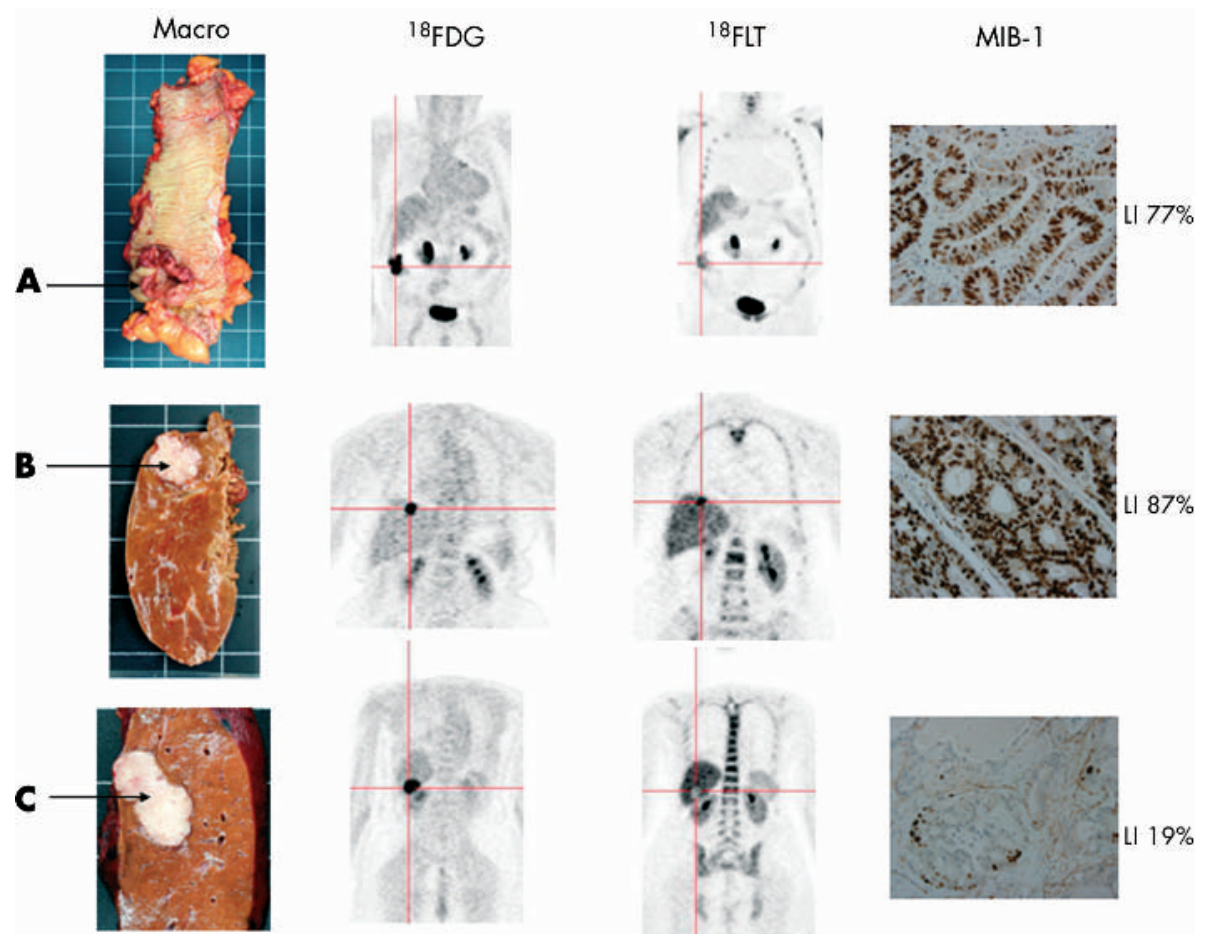

Figure 4 Primary tumour of the caecum $(A)$ and two liver metastases $(B, C)$ with their respective positron emission tomography images, with both ${ }^{18} \mathrm{~F}$ labelled 2-fluoro-2-deoxy-D-glucose $\left({ }^{18} \mathrm{FDG}\right)$ and ${ }^{18} \mathrm{~F}$ labelled 3 '-deoxy-3-fluorothymidine $\left({ }^{18} \mathrm{FLT}\right)$. Corresponding MIB-1 slides are shown at $\times 40$ power, with positive nuclei staining brown. The liver lesion (B) which stains very highly for MIB-1 (LI 87\%) is seen as avid for ${ }^{18} \mathrm{FLT}$ in comparison with the liver lesion (C) with a low MIB-1 stain (LI 19\%) which is not seen with ${ }^{18} \mathrm{FLT}$. The photopoenic appearance of the liver lesion (C) highlights the increased hepatic background activity secondary to glucuronidation of ${ }^{18} \mathrm{FLT}$ in contrast with the low uptake in the lesion by virtue of its low proliferative rate.

${ }^{18}$ FLT PET has recently been found in the investigation of solitary pulmonary nodules to be specific for malignant lesions. In addition, ${ }^{18}$ FLT correlates with the Ki-67 labelling index and estimation of prognosis. ${ }^{35}$ This hypothesis has been further validated by Vesselle and colleagues ${ }^{36}$ who assessed proliferation rates in 11 histologically proven non-small cell lung cancers using ${ }^{18}$ FLT PET and again demonstrated significant correlation with Ki-67.

The very nature of PET allows metabolic assessment of the whole tumour in its heterogeneic entirety. This coupled with the unique ability to quantitate the uptake of the tracers used, non-invasively and in vivo, has opened new avenues in the assessment of malignancy by functional imaging.

A system for grading the aggressiveness of CRC was first developed by Cuthbert Duke in 1932, ${ }^{37}$ and it was not until 1995 that a classification of prognostic markers was first suggested by the College of American Pathologists. ${ }^{38}$ Seventy years on, Dukes and TNM, along with the histological grade of the tumour, still remain the only reliable methods to dictate whether patients with CRC will receive adjuvant therapy. Unfortunately, under current guidelines, which have been set up in light of these prognostic indicators, there is still a cohort of patients who unnecessarily receive toxic chemotherapy while others who could potentially benefit from it are not recommended to receive it. In view of this, the prognostic value of Ki-67 in colorectal cancer has recently been revisited by the National Surgical Adjuvant Breast and Bowel Project Collaborative Study group in an attempt to define a marker on which therapeutic decisions could be made with greater precision. ${ }^{39}$

Ki-67 was selected for investigation because of its potential value in identifying populations of individuals who may or may not benefit from adjuvant chemotherapy. Significant prognostic value was found for patients with Dukes' B and C disease although it was unable to differentiate between groups who might benefit from adjuvant chemotherapy. Prior to this study, there have been contradictory reports regarding the role of $\mathrm{Ki}-67$ role as a prognostic indicator in CRC. Palmqvist and colleagues ${ }^{40}$ showed that those tumours with low Ki-67 expression at the invasive margin had a poor prognosis while Kimura and colleagues ${ }^{41}$ concluded that the tumours with high expression at the site of deepest invasion had a worse prognosis. In contrast, both Kubota and colleagues $^{42}$ who analysed 100 cases of CRC using Ki-67, and Kyzer and colleagues ${ }^{43}$ who examined 30 resected specimens of CRC, concluded that Ki-67 could not be used as a prognostic determinant in CRC.

Our study has demonstrated that ${ }^{18}$ FLT PET may be used as a non-invasive method of quantitating cellular proliferation in CRC. This may offer the opportunity for CRC to be imaged at a pretreatment stage for those undergoing surgery and perhaps define the population of patients who might benefit from neoadjuvant therapy. In addition, it may also help in identifying a group of patients with recurrent disease who would not benefit from firstline chemotherapy as a result of the low proliferation rate of the tumour, as demonstrated by the low SUV on ${ }^{18}$ FLT PET imaging.

This would offer the clinician a further prognostic marker with which to assess the aggressiveness of the disease and perhaps allow a more precise method for therapeutic decisions to be made. In real terms, a decision regarding response to chemotherapy in CRC is usually judged six weeks into a 12 week treatment cycle. The potential of avoiding six weeks of unnecessary toxic chemotherapy by differentiating the non-responders from the responders at a pretreatment stage is a prospect which cannot be ignored.

Future work will help in validating ${ }^{18}$ FLT PET as a potential tool for the non-invasive in vivo assessment of CRC and may 
enable more accurate evaluation of the antitumoral effects of new as well as established anticancer drug treatments aimed at competing with DNA synthesis.

\section{ACKNOWLEDGEMENTS}

The authors wish to acknowledge Ian Croasdale and Caroline Townsend for their excellent radiographic and technical support in the imaging of the patients used in this study, and Phillipa Munson for her help in performing the immunohistochemistry.

\section{Authors' affiliations}

D L Francis, Institute of Nuclear Medicine, and Department of Surgery, Royal Free and University College Medical School, Middlesex Hospital, London, UK

A Freeman, M Novelli, Department of Histopathology, Royal Free and University College Medical School, Middlesex Hospital, London, UK

D Visvikis, D C Costa, P J Ell, Institute of Nuclear Medicine, Royal Free and University College Medical School, Middlesex Hospital, London, UK S K Luthra, IRSL Cyclotron Unit, Hammersmith Hospital, London, UK I Taylor, Department of Surgery, Royal Free and University College Medical School, Middlesex Hospital, London, UK

\section{REFERENCES}

1 Beets G, Penninckx F, Schiepers C, et al. Clinical value of whole-body positron emission tomography with[18F]fluorodeoxyglucose in recurrent colorectal cancer. Br J Surg 1994;81:1666-70.

2 Vitola JV, Delbeke D, Sandler MP, et al. Positron emission tomography to stage suspected metastatic colorectal carcinoma to the liver. Am J Surg 1996;171:21-6.

3 Delbeke D, Vitola JV, Sandler MP, et al. Staging recurrent metastatic colorectal carcinoma with PET. J Nucl Med 1997;38:1 196-201.

4 Lai DT, Fulham M, Stephen MS, ef al. The role of whole-body positron emission tomography with[18F]fluorodeoxyglucose in identifying operable colorectal cancer metastases to the liver. Arch Surg 1996;131:703-7.

5 Ogunbiyi OA, Flanagan FL, Dehdashti F, et al. Detection of recurrent and metastatic colorectal cancer: comparison of positron emission tomography and computed tomography. Ann Surg Oncol 1997:4:613-20.

6 Valk PE, Abella-Columna E, Haseman MK, ef al. Whole-body PET imaging with[18F]fluorodeoxyglucose in management of recurrent colorectal cancer. Arch Surg 1999;134:503-11.

7 Flamen P, Stroobants S, Van Cutsem E, et al. Additional value of whole-body positron emission tomography with fluorine-18-2-fluoro-2-deoxy-D-glucose in recurrent colorectal cancer. J Clin Oncol 1999;17:894-901.

8 Staib L, Schirrmeister H, Reske SN, et al. Is (18)F-fluorodeoxyglucose positron emission tomography in recurrent colorectal cancer a contribution to surgical decision making? Am J Surg 2000;180:1-5.

9 Arulampalam T, Costa D, Visvikis D, et al. The impact of FDG-PET on the management algorithm for recurrent colorectal cancer. Eur J Nucl Med 2001;28:1758-65.

10 Mankoff DA, Dehdashti F, Shields AF. Characterizing tumors using metabolic imaging: PET imaging of cellular proliferation and steroid receptors. Neoplasia 2000;2:71-88.

11 Eary JF, Mankoff DA, Spence AM, et al. 2-[C-11]thymidine imaging of malignant brain tumors. Cancer Res 1999;59:615-21.

12 Martiat P, Ferrant A, Labar D, et al. In vivo measurement of carbon-11 thymidine uptake in non-Hodgkin's lymphoma using positron emission tomography. J Nucl Med 1988;29:1633-7.

13 Shields AF, Mankoff DA, Link JM, et al. Carbon-11-thymidine and FDG to measure therapy response. J Nucl Med 1998;39:1757-62.

14 Vander BT, Pauwels S, Lambotte L, et al. Brain fumor imaging with PET and 2[carbon-11] thymidine. J Nucl Med 1994;35:974-82.

15 van Eijkeren ME, De Schryver A, Goethals P, et al. Measurement of short-term $11 \mathrm{C}$-thymidine activity in human head and neck tumours using positron emission tomography (PET). Acta Oncol 1992;31:539-43.

16 Krohn KA, Mankoff DA, Eary JF. Imaging cellular proliferation as a measure of response to therapy. J Clin Pharmacol 2001;(suppl):96S-103S.

17 Ahnen DJ, Feigl P, Quan G, et al. Ki-ras mutation and p53 overexpression predict the clinical behavior of colorectal cancer: a Southwest Oncology Group study. Cancer Res 1998;58:1149-58.
18 Witzig TE, Loprinzi CL, Gonchoroff NJ, et al. DNA ploidy and cell kinetic measurements as predictors of recurrence and survival in stages B2 and C colorectal adenocarcinoma. Cancer 1991;68:879-88.

19 Venkatesh KS, Weingart DJ, Ramanujam PJ. Comparison of double and single parameters in DNA analysis for staging and as a prognostic indicator in patients with colon and rectal carcinoma. Dis Colon Rectum 1994;37:1142-7

20 Brown DC, Gatter KC. Ki67 protein: the immaculate deception? Histopathology 2002:40:2-11

21 Higashi K, Clavo AC, Wahl RL. Does FDG uptake measure proliferative activity of human cancer cells? In vitro comparison with DNA flow cytometry and tritiated thymidine uptake. J Nucl Med 1993;34:414-19.

22 Buck AC, Schirrmeister HH, Guhlmann CA, et al. Ki-67 immunostaining in pancreatic cancer and chronic active pancreatitis: does in vivo FDG uptake correlate with proliferative activity? J Nucl Med 2001;42:721-5.

23 Grierson JR, Shields AF. Radiosynthesis of 3'-deoxy-3'[(18)F]fluorothymidine: [(18)F]FLT for imaging of cellular proliferation in vivo. Nucl Med Biol 2000;27:143-56.

24 Munch-Petersen B, Cloos L, Jensen HK, et al. Human thymidine kinase 1. Regulation in normal and malignant cells. Adv Enzyme Regul 1995;35:69-89.

25 Broet $\mathbf{P}$, Romain S, Daver A, et al. Thymidine kinase as a proliferative marker: clinical relevance in 1,692 primary breast cancer patients. J Clin Oncol $2001 ; 19: 2778-87$

26 Rasey JS, Grierson JR, Wiens LW, et al. Validation of FLT uptake as a measure of thymidine kinase-1 activity in A549 carcinoma cells. J Nud Med 2002;43:1210-17

27 Hamacher K, Coenen HH, Stocklin G. Efficient stereospecific synthesis of nocarrier-added 2-[18F]-fluoro-2-deoxy-D-glucose using aminopolyether supported nucleophilic substitution. J Nucl Med 1986;27:235-8.

28 Cleij MC, Steel CJ, Brady F, et al. An improved synthesis of 3-deoxy-3[18F]Fluorothymidine. J Lab Comp Radioph 2001;44:S871.

29 Visvikis D, Francis DL, Costa DC, et al. Quantitative comparison of 18FDG and 18FLT in colorectal carcinoma. Eur J Nucl Med 2002;29:S79.

30 Visvikis D, Costa DC, Croasdale I, et al. CT-based attenuation correction in the calculation of semi-quantitative indices off(18)F]FDG uptake in PET. Eur J Nucl Med Mol Imaging 2003;30:344-53.

31 Francis DL, Visvikis D, Costa DC, et al. Potential impact of[(18)F]3'-deoxy-3' fluorothymidine versus[(18)F]fluoro-2-deoxy- D-glucose in positron emission tomography for colorectal cancer. Eur J Nucl Med Mol Imaging 2003;30:988-94.

32 Tahara T, Ichiya Y, Kuwabara Y, et al. High[18F]-fluorodeoxyglucose uptake in abdominal abscesses: a PET study. J Comput Assist Tomogr 1989;13:829-31

33 Bakheet SM, Powe J, Kandil A, et al. F-18 FDG uptake in breast infection and inflammation. Clin Nucl Med 2000;25:100-3.

34 Larson SM. Cancer or inflammation? A Holy Grail for nuclear medicine. J Nud Med 1994;35: 1653-5.

35 Buck AK, Schirrmeister H, Hetzel M, et al. 3-deoxy-3-[(18)F]fluorothymidinepositron emission tomography for noninvasive assessment of proliferation in pulmonary nodules. Cancer Res 2002;62:3331-4.

36 Vesselle H, Grierson J, Muzi M, et al. In vivo validation of 3'deoxy-3'$[(18) F]$ fluorothymidine ([(18)F]FLT) as a proliferation imaging tracer in humans: Correlation of $[(18) F]$ FLT uptake by positron emission tomography with $\mathrm{Ki}-67$ immunohistochemistry and flow cytometry in human lung tumors. Clin Cancer Res 2002:8:3315-23.

37 Dukes CE. The classification of cancer of the rectum. J Pathol 1932;35:323.

38 Henson DE, Hutter RV, Sobin LH, et al. Protocol for the examination of specimens removed from patients with colorectal carcinoma. A basis for checklists. Cancer Committee, College of American Pathologists. Task Force for Protocols on the Examination of Specimens from Patients with Colorectal Cancer. Arch Pathol Lab Med 1994;1 18:122-5.

39 Allegra CJ, Paik S, Colangelo LH, et al. Prognostic value of thymidylate synthase, $\mathrm{Ki}-67$, and p53 in patients with Dukes' $B$ and $C$ colon cancer: a National Cancer Institute-National Surgical Adjuvant Breast and Bowel Project collaborative study. J Clin Oncol 2003;21:241-50.

40 Palmqvist R, Sellberg P, Oberg A, et al. Low tumour cell proliferation at the invasive margin is associated with a poor prognosis in Dukes' stage B colorectal cancers. Br J Cancer 1999:79:577-81.

41 Kimura T, Tanaka S, Haruma K, et al. Clinical significance of $\mathrm{MUCl}$ and Ecadherin expression, cellular proliferation, and angiogenesis at the deepest invasive portion of colorectal cancer. Int J Oncol 2000; 16:55-64.

42 Kubota Y, Petras RE, Easley KA, et al. Ki-67-determined growth fraction versus standard staging and grading parameters in colorectal carcinoma. A multivariate analysis. Cancer 1992;70:2602-9.

43 Kyzer S, Gordon PH. Determination of proliferative activity in colorectal carcinoma using monoclonal antibody Ki67. Dis Colon Rectum 1997;40:322-5. 